\section{Migrations cellulaires collectives}

Éric Theveneau ${ }^{1}$, Nicolas David ${ }^{2,3}$

$>$ L'étude de la migration cellulaire, d'abord focalisée sur les mécanismes régissant la motilité au niveau d'une cellule unique, incorpore désormais les phénomènes coopératifs à l'échelle d'un groupe de cellules. Ces migrations dites collectives se produisent dans des contextes physiologiques ou pathologiques. Dans cette synthèse, nous discuterons les points qui permettent de définir le caractère collectif d'une migration. Nous aborderons les principaux concepts régissant les migrations collectives de tissus épithéliaux et mésenchymateux, et, plus particulièrement, les mécanismes régulant la polarité et orientant la direction des cellules. <

Les migrations cellulaires sont essentielles au développement embryonnaire - elles permettent l'organisation des cellules en organes -, à l'homéostasie des tissus (cicatrisation) et à l'immunité [1]. En plus de ces rôles physiologiques, les migrations cellulaires sont également impliquées dans diverses situations pathologiques comme la formation de métastases cancéreuses [2].

Les mécanismes assurant le déplacement d'une cellule unique ont été largement analysés [3]. Schématiquement, la cellule commence par émettre une extension cytoplasmique (filopode, lamellipode, pseudopode) sous l'effet de la polymérisation de l'actine corticale qui déforme la membrane plasmique. Une fois formée, cette extension s'ancre au substrat. Si ce dernier est une matrice extracellulaire, l'ancrage est assuré par des adhérences focales, dans lesquelles des protéines comme les intégrines et le syndécan ${ }^{1}$ relient le cytosquelette à la matrice [4]. Dans le cas où le substrat est une autre cellule, l'ancrage s'effectue grâce à des molécules d'adhérence cellule-cellule comme les cadhérines. Ensuite, la contraction du cytosquelette

\footnotetext{
${ }^{1}$ Le syndécan est un protéoglycane composé d'une core protéine qui porte des chaînes de glycosaminoglycanes; transmembranaire, son domaine cytoplasmique est très court, et interagit avec des protéines du cytosquelette ou des voies de signalisation.
}

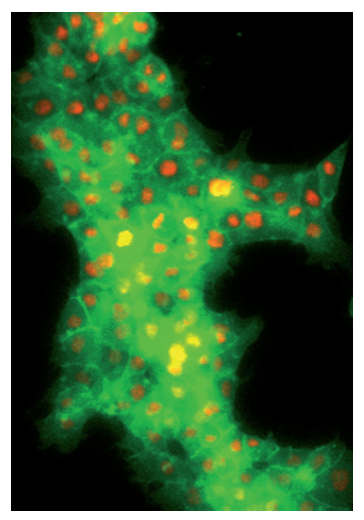

${ }^{1}$ Centre de biologie du développement, unité mixte de recherche 5547, CNRS, université Paul Sabatier; 118, route de Narbonne, 31400 Toulouse, France; ${ }^{2}$ Inserm U1024, CNRS unité mixte de recherche 8197, F-75005 Paris, France ;

${ }^{3}$ École normale supérieure (ENS), institut de biologie de l'ENS (IBENS), F-75230 Paris, France. eric.theveneau@univ-tlse3.fr ndavid@biologie.ens.fr

d'actomyosine assure le déplacement cellulaire par traction sur les adhérences focales et rétraction de l'arrière de la cellule (qui, au contraire, se détache du substrat) [3,4]. Afin d'être efficaces, ces processus doivent être polarisés. Dans la cellule, cette polarité est essentiellement contrôlée par les petites GTPases de la famille Rho, Racl, Cdc42 et RhoA, qui régulent la dynamique du cytosquelette. Au front de migration, Racl et Cdc42 favorisent la formation des protrusions, alors qu'à l'arrière, RhoA induit la formation de fibres de stress assurant la contractilité cellulaire. Racl et RhoA sont mutuellement antagonistes et une polarité peut donc émerger spontanément au sein de la cellule. Néanmoins, lors des migrations orientées de cellules isolées, cette polarité est souvent maintenue par des signaux de guidage externes, distribués en gradients locaux.

Les grands principes régissant la migration à l'échelle de la cellule sont aujourd'hui bien compris. Cependant, il existe de nombreuses variations en fonction des types cellulaires étudiés, et les mécanismes moléculaires expliquant ces différences sont, eux, moins bien connus. Dans les organismes pluricellulaires vient s'ajouter un degré de complexité supplémentaire. En effet, à l'exception de ceux de certaines cellules comme les lymphocytes ou les cellules germinales, la plupart des déplacements se font sous forme d'ensemble de cellules et non de cellules solitaires $[5,6]$. C'est le cas lors de la migration de feuillets épithéliaux, notamment au cours de la cicatrisation, mais aussi lors de la migration de groupes de cellules, qu'elles soient épithéliales, endothéliales ou mésenchymateuses, lors du développement et de l'organogenèse [7, 8]. Enfin, un certain nombre de tumeurs migrent collectivement pour envahir les tissus environnants [9]. Le fait de migrer en groupe implique des mécanismes spécifiques et permet l'émergence de propriétés nouvelles. Nous discutons ici ces spécificités, mises en lumière au cours de la décennie. 
A Transplantation de cellules

Embryon de poisson zèbre, plaque préchordale

Embryon A Embryon B

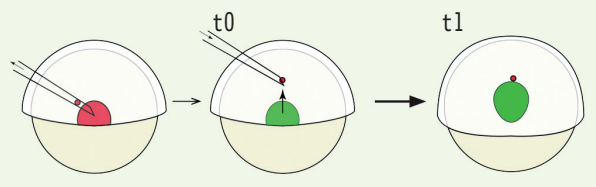

Embryon de xénope, cellules de la crête neurale

Embryon A Embryon B

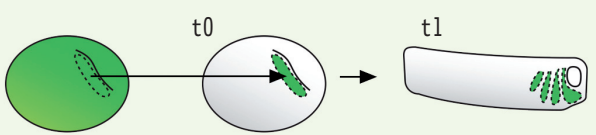

B Mise en culture

Embryon de xénope, cellules de la crête neurale
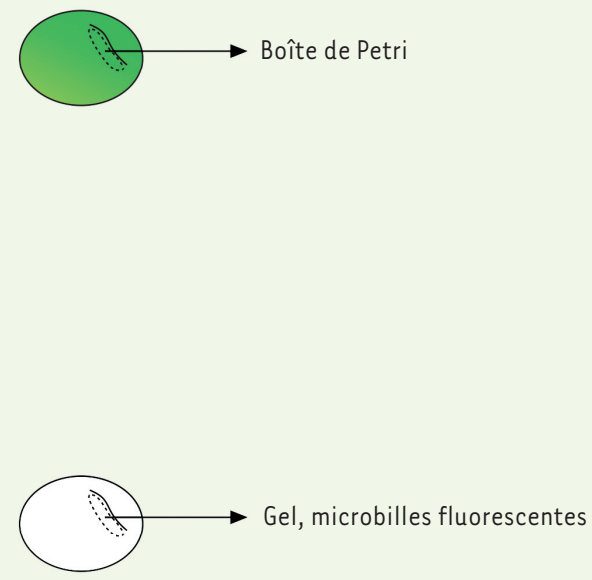

Cellule isolée

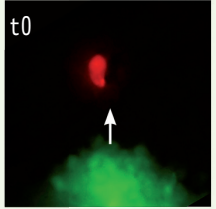

Marquage mosaïque

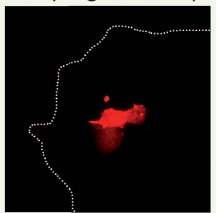

Marquage mosaïque
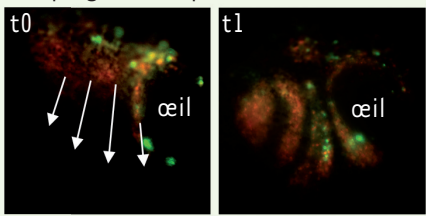

Marquage membranaire mosaïque
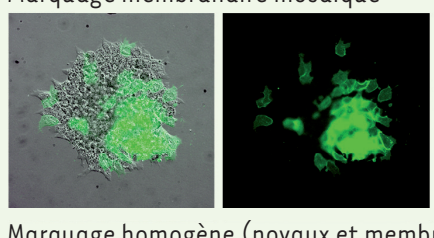

Marquage homogène (noyaux et membranes)
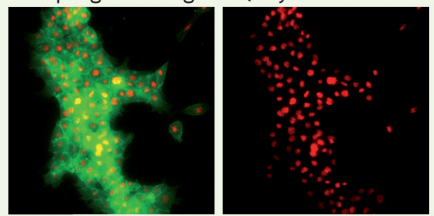

Cellules non marquées cultivées sur ge
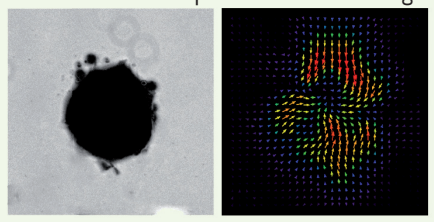

Figure 1. Exemples de modèles et stratégies permettant l'analyse des mouvements collectifs. A. Transplantation de cellules. Les cellules transplantées en dehors du groupe permettent de comparer le comportement des cellules isolées avec celui des cellules du groupe. Les marquages mosaïques facilitent le suivi d'une cellule au sein d'un groupe. B. Mise en culture. Les marquages mosaïques permettent un meilleur suivi de la dynamique membranaire parfois difficile in vivo. Les marquages nucléaires facilitent le traçage cellulaire et l'extraction de paramètres tels que la vitesse, la vélocité, la direction et la coordination, notamment dans le cadre d'un test de réponse à un gradient. Les cellules peuvent être déposées sur des gels contenant des billes fluorescentes. L'analyse du déplacement des billes permet de calculer les forces de traction exercées par les cellules et, par extension, d'en extraire la force des contacts cellules-cellules au sein du groupe épithélial (voir les travaux de Fredberg et Trepat [43]).

\section{Du collectif et de l'individuel}

Avant de discuter des mécanismes régissant les migrations cellulaires collectives, il convient de s'entendre sur la définition d'une migration collective. Pendant longtemps, ce terme a été réservé aux migrations de cellules épithéliales fortement adhérentes les unes aux autres. Plus récemment, une définition plus large a été proposée, et c'est celle-ci que nous adoptons ici. Cette définition repose sur deux critères. Premièrement, les cellules doivent se déplacer ensemble. Autrement dit, le mouvement de chaque cellule est corrélé au mouvement global de la population. Deuxièmement, les interactions entre cellules au sein du groupe affectent leur trajectoire. Cela distingue un processus réellement collectif d'un groupe de cellules qui migreraient toutes de la même façon au même moment, mais de façon indépendante les unes des autres. Ce second critère est plus difficile à établir. Le principe expérimental est d'isoler une cellule ou un petit sousensemble de cellules. Ceci peut être fait par ablation d'une partie du groupe en migration, ou par transplantation de cellules hors du groupe (Figure I). Ensuite, on analyse le comportement de la ou des cellules isolées pour déterminer s'il se trouve modifié par l'absence des cellules voisines.

\section{Interactions entre les cellules}

Le point clé d'une migration collective est que les cellules qui se déplacent s'influencent mutuellement. Une des composantes importantes de cette coopération fait intervenir un contact physique entre les cellules. Dans les épithéliums, les cellules sont attachées de façon 


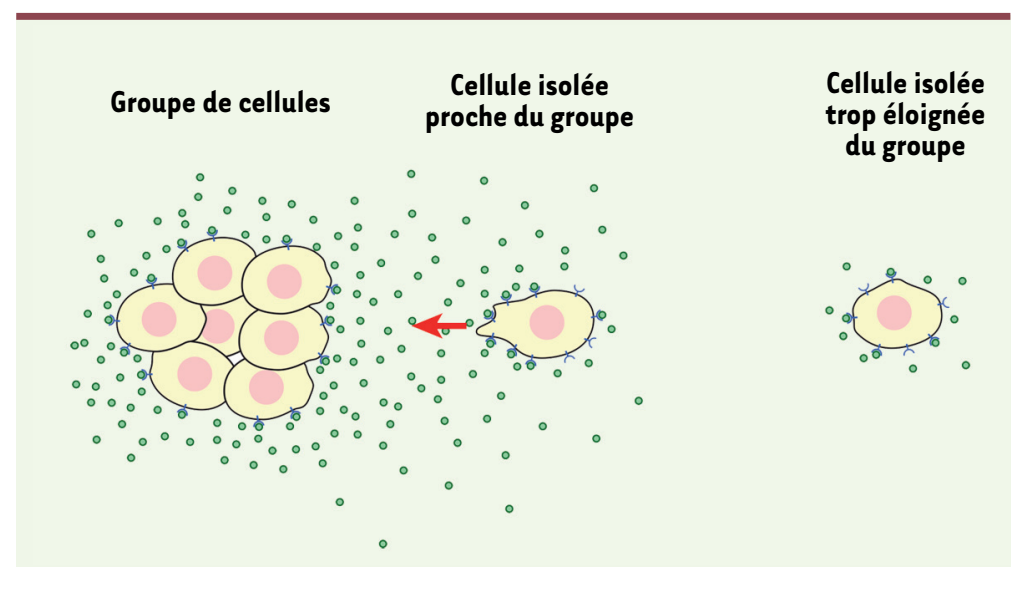

Figure 2. Principe de l'attraction mutuelle ou co-attraction. Toutes les cellules sécrètent un chimioattractant (- billes vertes) dont elles expriment également le récepteur (Y bleu). Les zones de forte densité cellulaire agissent comme un centre de gravité qui agrège progressivement les cellules isolées avoisinantes. Si une cellule sort du gradient local produit par le groupe, elle ne sera plus soumise à la coattraction. relativement stable par des jonctions intercellulaires adhérentes serrées et des desmosomes qui impliquent respectivement des protéines transmembranaires telles que les cadhérines, les claudines et les desmogléines (ou desmocollines). Les épithéliums présentent donc une certaine rigidité structurale, et l'organisation du tissu au cours de la migration varie peu. Ainsi, lors de la migration de très petits groupes comme les cellules de bordure de drosophile, aucun changement de position relative des cellules n'est observé [8]. Le groupe tourne sur lui-même, la cellule au front peut donc changer, mais l'organisation du groupe est conservée. Dans des structures épithéliales plus importantes, comme une monocouche de cellules en culture, on peut néanmoins constater un certain degré de réorganisation au cours de la migration [10].

L'une des propriétés intéressantes des protéines de jonctions intercellulaires est que celles-ci forment des complexes macromoléculaires qui s'attachent au cytosquelette et organisent ainsi ce dernier en un réseau supracellulaire. Cela entraîne un couplage mécanique important à l'échelle de la population et favorise l'émergence d'une contractilité tissulaire plus que cellulaire. Dès lors, toutes les cellules n'exercent pas systématiquement de forces de traction sur la matrice lorsque que le tissu se déplace. Les mesures effectuées sur les monocouches épithéliales montrent que les zones actives en termes de traction sur la matrice apparaissent sous la forme d'îlots de plusieurs diamètres cellulaires $[11,12]$. Ceux-ci se comportent comme une supercellule dont la taille varie au cours du temps et peuvent exercer sur la matrice une force supérieure à celle que produit une cellule individuelle. Ces îlots actifs sont entourés de cellules transitoirement passives, mais qui se déplacent néanmoins grâce à leur attachement fort aux autres cellules.

Dans les tissus mésenchymateux, l'essentiel des jonctions cellulecellule sont des jonctions adhérentes impliquant des cadhérines [13]. Ces jonctions ne sont pas stables au cours du temps, ce qui permet une plus grande plasticité et une plus grande liberté de mouvement des cellules au sein de la population. Ainsi, des cellules initialement voisines peuvent se retrouver très éloignées les unes des autres après quelques heures de migration. C'est le cas par exemple lors de la colonisation du tube digestif par les crêtes neurales entériques [14]. Mais il arrive également que les relations spatiales entre cellules soient globalement conservées au cours du temps, comme dans la migration du mésoderme [15] ou des crêtes neurales céphaliques [16].

De façon remarquable, il a été récemment établi que les mécanismes de maintien de la cohésion du groupe ne se limitent pas aux jonctions physiques entre cellules, mais incluent également des interactions chimiques à distance [17]. Les crêtes neurales de xénope expriment le chimioattractant $\mathrm{C} 3 \mathrm{a}$, ce qui crée un gradient de C3a depuis le centre du groupe vers l'extérieur. Or, les cellules de crêtes neurales expriment également le récepteur du C3a. De ce fait, si une cellule tend à s'éloigner des autres, elle est attirée vers la zone de plus forte concentration en C3a, c'est-à-dire vers le groupe de cellules $[17,40]$. Ce mécanisme, basé sur un chimiotactisme paracrine et baptisé coattraction, permet donc aux cellules de rester ensemble au cours de leur migration (Figure 2). Un tel mécanisme existe vraisemblablement dans d'autres systèmes collectifs mésenchymateux, même si cela reste à établir. On retrouve en tout cas un système équivalent chez les bactéries telles qu'Escherichia coli, qui sécrètent un facteur leur permettant de voyager sous la forme d'un essaim le long d'un gradient de nourriture [18], et chez l'amibe sociale Dictyostélium discoideum [19]. Lors d'une phase de carence, les amibes s'attirent mutuellement en secrétant de I'AMP cyclique. Elles s'agrègent alors progressivement en une structure pluricellulaire, le pseudo-plasmode, qui leur permet de résister plusieurs jours pendant la recherche de conditions favorables.

\section{Polarité et information de direction}

Comme nous l'avons mentionné dans l'introduction, le guidage d'une cellule isolée nécessite que sa polarité soit orientée et maintenue par un gradient local (chimioattractant, rigidité du substrat, etc.). De même, le guidage d'un groupe de cellules requiert une information de 
Épithélium

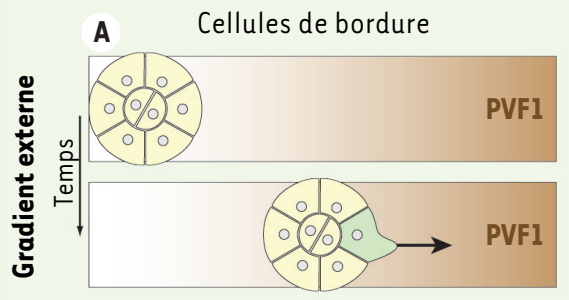

C Primordium de la ligne latérale

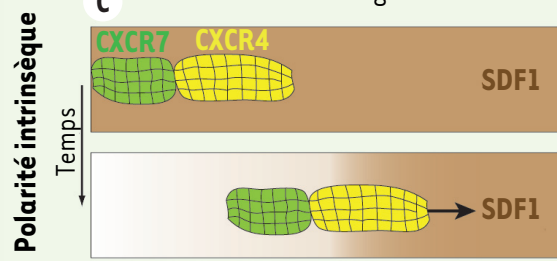

Mésenchyme

B Cellules de la crête neurale

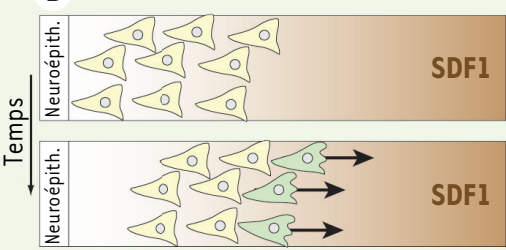

D Plaque préchordale

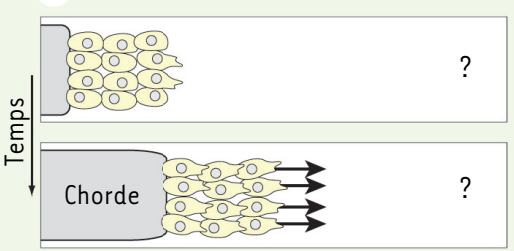

B'

Inhibition de contact/polarité

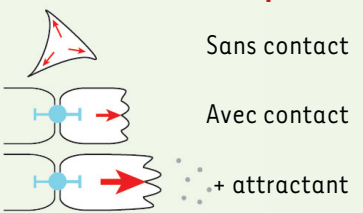

D'

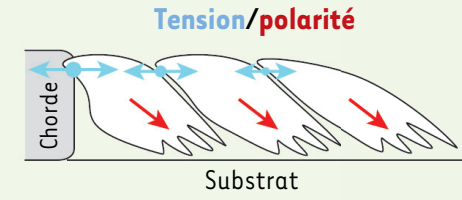

Figure 3. Polarité et information de direction. A. Cellules de bordure de drosophile. Le groupe est polarisé par l'émergence d'une cellule pionnière (vert) favorisée par la présence d'un gradient externe. B-B'. Cellules de la crête neurale. Le mouvement est déclenché par l'asymétrie du système avec un bord libre d'un côté. Ensuite, chaque cellule est polarisée par le contact avec ses voisines, seules les cellules déjà polarisées voient leur polarité modulée par le gradient externe. C. Primordium de la ligne latérale. Le groupe possède une polarité intrinsèque. Le gradient n'existe pas a priori et est créé in situ par endocytose préférentielle à l'arrière du groupe. D-D'. Plaque préchordale. Le mouvement est induit par l'asymétrie du système avec un bord libre d’un côté. La polarité de chaque cellule est induite et maintenue par les tensions entre cellules générées par le mouvement du groupe.

direction. Néanmoins, la collectivité permet l'émergence de propriétés nouvelles et de modes de guidage différents de ceux existants pour les cellules seules.

\section{Interprétation d'un gradient à l'échelle supracellulaire}

Les cellules de bordure de drosophile traversent la chambre ovarienne en utilisant plusieurs gradients locaux partiellement redondants. Les cellules expriment PVR (PDGF-VEGF receptor), et EGFR (epidermal growth factor receptor) qui se lient respectivement au PVFl (PDGFVEGF-related factor 1) et à Spitz, Keren et Gurken [8]. Lors de la première phase de migration, le guidage est assuré, comme dans des cellules isolées, par l'activation de Racl au front des cellules, qui polarise chaque cellule individuellement. En revanche, pendant la seconde partie de leur mouvement, il semble que la polarisation soit assurée à l'échelle supracellulaire. Au sein de chaque cellule, le niveau d'activation de Racl est homogène. Cependant, les niveaux diffèrent entre une cellule dite pionnière et les autres cellules (Figure 3A). C'est cette différence de niveau de signalisation entre cellules qui polarise le groupe et lui donne la direction du mouvement $[20,21,40](\rightarrow)$. Les mécanismes contrôlant cette asymétrie à l'échelle du groupe restent mal compris, mais ils impliquent une régulation de la de Myriam Reffay et al., page 736 de ce numéro $(\rightarrow)$ Voir la Nouvelle dynamique de l'actine. Ceci passe par l'activation de la moésine, dans un processus dépendant de Rabll qui permet de concentrer Racl dans les cellules au front de migration [22, 23]. Parl (protease activated receptor 1 ) joue également un rôle sur la contraction du cytosque- lette d'actomyosine [24]. Parl se localise à l'arrière du groupe où il inhibe la phosphatase de la myosine II, ce qui favorise une forte activité contractile et la rétraction des cellules.

\section{Renforcement d'une polarité existante via un gradient externe}

Les cellules de la crête neurale céphalique migrent collectivement sous la forme d'un mésenchyme [41]. Dans cette population, la polarité cellulaire est induite par les contacts cellule-cellule, et non par un signal externe [25]. Ces contacts entraînent une réponse appelée inhibition de locomotion par contact (contact-inhibition of locomotion) [26]. Brièvement, lors d'un contact physique entre deux cellules, une jonction cellulaire transitoire contenant la $\mathrm{N}$-cadhérine se forme et active la voie de polarité planaire de la voie Wnt (Wnt-planar cell polarity pathway). En aval, RhoA est activée et Racl inhibée. Cela promeut une rétraction des protrusions [26]. Dès lors, les cellules sont polarisées selon un axe contact cellulaire-bord libre (Figure 1B). Seules les cellules présentant cette polarité peuvent répondre à l'attractant externe, le SDFl (stromal cell-derived factor-1), qui, lui, ne fait qu'amplifier et stabiliser cette polarité déjà formée (Figure $3 B-B^{\prime}$ ), sans être capable de l'induire de novo [25]. Cet effet de groupe, 
dépendant des interactions cellulaires, est aussi observé lors de la réponse à des signaux négatifs [27].

\section{Polarité intrinsèque au groupe migratoire organisant un signal initialement homogène}

La collectivité permet à certains groupes de s'affranchir d'un signal de direction externe. C'est le cas lors de la migration du primordium de la ligne latérale chez le poisson [28]. Ce groupe d'environ 200 cellules migre de la tête vers la queue du poisson le long d'un rail de SDFl. Cependant, ce rail, s'il définit le trajet du primordium, ne porte pas d'information de direction. Expérimentalement, le primordium peut d'ailleurs l'emprunter indifféremment dans un sens ou l'autre. La direction de la migration est ici définie par l'asymétrie du primordium lui-même (Figure 3C). En effet, la moitié antérieure du primordium exprime le récepteur $C x c r 4 b$, qui assure la réponse des cellules à Sdfl. La moitié postérieure exprime en revanche le récepteur $\mathrm{Cxcr} 7$. Celui-ci ne provoque pas de réponse cellulaire, mais induit l'internalisation rapide de Sdfl dans la partie postérieure du primordium, créant ainsi un gradient local de chimiokine qui oriente alors la migration $[29,30]$.

\section{Auto-organisation d'un groupe en absence de signal externe}

Lors de la cicatrisation d'un feuillet épithélial, la migration est déclenchée en réponse à l'apparition d'un bord libre. Cela commence par une augmentation de la motilité des cellules situées à proximité de l'espace à combler. Certaines adoptent même un phénotype très polarisé et se placent à l'avant de longues digitations envahissant la blessure [31], sans que l'on sache comment cette interruption de l'épithélium induit la migration $(\rightarrow)[40$, 41]. Dans ces systèmes, les cellules tendent à aligner progressivement leurs polarités individuelles avec le mouvement général du tissu [12, $(\rightarrow)$ Voir la Nouvelle de Myriam Reffay et al., page 736 de ce numéro
32]. Cela aboutit à une diminution progressive des stress locaux aux interfaces entre cellules, et favorise un mouvement cohérent du feuillet. On passe donc rapidement d'un système chaotique réagissant à l'apparition de cette blessure à une structure s'autoorganisant sur la base des contraintes mécaniques imposées par le mouvement général. L'importance des interactions locales sur l'alignement du mouvement des cellules d'un groupe permettant la transition vers un déplacement collectif a également été modélisée avec succès [33].

La migration de la plaque préchordale constitue sans doute un autre exemple de groupe s'orientant indépendamment de signaux externes (Figure 3D). L'information de direction semble être portée par le groupe de cellules et ne pas être fournie par l'environnement [34]. Si la nature de cette information reste à déterminer, une hypothèse attrayante est qu'elle soit également de nature mécanique. En effet, une tension appliquée via les jonctions adhérentes est suffisante pour polariser une cellule de plaque préchordale et induire sa migration [15]. L'orientation du groupe proviendrait alors de l'existence d'un gradient de tensions entre son bord antérieur libre de toute contrainte, et son bord postérieur attaché aux cellules de notochorde.

\section{Discussion et perspectives}

Pourquoi migrer collectivement? Peut-être est-ce une nécessité fonctionnelle. C'est le cas lors de la cicatrisation d'un épithélium qui doit à la fois maintenir son rôle de barrière et se déplacer pour refermer la blessure. Au cours du développement, cela permet de maintenir ensemble des cellules appelées à participer à une même structure. Enfin, un groupe de cellules peut aussi assurer le déplacement passif de cellules incapables de migrer, comme les cellules polaires portées par les cellules de bordure.

La collectivité permet l'émergence de propriétés nouvelles. Elle peut améliorer la détection de faibles gradients de chimiokines, en autorisant la lecture du gradient sur une plus grande distance (lecture par le groupe au lieu d'une cellule unique). La collectivité permet même de s'affranchir de signaux de guidage externes, l'orientation est alors assurée par la polarité interne du groupe. Une possibilité intéressante est que les mécanismes collectifs assurent une plus grande robustesse du système, les décisions étant prises par et moyennées sur un ensemble de cellules et non par des cellules isolées. À ce titre, il est intéressant de constater que dans certains groupes, certaines compétences, comme la capacité à lire un gradient de molécule de guidage et à y répondre, ont été transférées de la cellule vers le groupe. Ceci réduit l'autonomie des cellules individuelles, et pourrait limiter le risque de les voir s'égarer.

De façon remarquable, les grands principes d'interaction et de coopération décrits ici entre cellules d'une même population existent aussi à des échelles supérieures. De telles coopérations ont notamment été observées entre différents types cellulaires, que ce soit lors du développement embryonnaire [35] ou entre des cellules cancéreuses et des fibroblastes [36, 42]. À une échelle plus grande encore, les déplacements collectifs d'animaux sont aussi régis par des mécanismes similaires tels que l'attraction mutuelle ou la répulsion par contact physique $[37,38]$. Une approche de type biologie des systèmes, allant des molécules aux populations, permettra de mieux comprendre l'émergence de nouvelles aptitudes basées sur la coopération entre éléments de réseaux dynamiques, qu'il s'agisse de cellules, tissus ou animaux. Notamment, de nombreux outils mathématiques et statistiques développés pour modéliser les comportements animaliers peuvent être facilement adaptés à la modélisation des populations cellulaires. L'apport de cette biologie prédictive in silico est un complément précieux à la recherche expérimentale. $\diamond$ 


\section{GLOSSAIRE}

\section{Cellules de bordure}

Groupe épithélial d'environ huit cellules, émanant de l'épithélium folliculaire antérieur de la chambre ovarienne de drosophile, qui migrent postérieurement vers l'ovocyte. Ces cellules sont requises pour la mise en place de l'axe antéro-postérieur et la formation du micropyle, nécessaire pour la fécondation.

Cellules de la crête neurale

Cellules mésenchymateuses qui, en fin de neurulation, migrent depuis la partie dorsale du neuroepithélium et colonisent l'ensemble de l'embryon avant de se différencier en os, cartilage, cellules gliales, neurones ou mélanocytes.

\section{Plaque préchordale}

Groupe de cellules mésenchymateuses, situées en avant de la notochorde, qui migrent sous l'ectoderme vers la future région céphalique au cours de la gastrulation.

\section{Primordium de la ligne latérale}

Groupe d'environ 200 cellules épithéliales qui migrent de la tête vers la queue des têtards ou alevins. Les cellules déposent dans leur sillage cinq à sept neuromastes, organes sensoriels de détection des mouvements d'eau.

\section{SUMMARY}

\section{Collective cell migrations}

Historically centered on the study of individual cell motility, the field of cell migration has recently moved up one level to look at cooperative behaviour within migratory cell populations. It is now well established that numerous physiological and pathological migration events involve collectively migrating cells rather than solitary cells or concomitantly migrating individual cells. In this review, we first discuss the criteria allowing a given migratory event to be classified as collective cell migration. We then summarize the main concepts that rule collective cell migration in epithelial and mesenchymal tissues with a main focus on mechanisms controlling polarity and directionality in cell collectives. $\diamond$

\section{LIENS D'INTÉRÊT}

Les auteurs déclarent n'avoir aucun lien d'intérêt concernant les données publiées dans cet article.

\section{REMERCIEMENTS}

E.T. est soutenu par le CNRS, l'université Paul Sabatier, la Fondation pour la recherche médicale (AJE201224) et la région Midi-Pyrénées (programme accueil de nouvelles équipes d'excellence). N.D. est soutenu par le CNRS, financé par l'Association pour la recherche contre le cancer (ARC SFI20111203770 et ARC PJA20131200143).

\section{RÉFÉRENCES}

1. Ilina 0, Friedl P. Mechanisms of collective cell migration at a glance. J Cell Sci 2009 ; 122 : 3203-8.

2. Bravo-Cordero J), Hodgson L, Condeelis J. Directed cell invasion and migration during metastasis. Curr Opin Cell Biol $2012 ; 24: 277-83$.

3. Ridley AJ. Life at the leading edge. Cell $2011 ; 145$ : 1012-22.
4. Wehrle-Haller B. Assembly and disassembly of cell matrix adhesions. Curr Opin Cell Biol $2012 ; 24$ : 569-81.

5. Rorth P. Collective cell migration. Annu Rev Cell Dev Biol 2009 ; 25 : 407-29.

6. Friedl P, Gilmour D. Collective cell migration in morphogenesis, regeneration and cancer. Nat Rev Mol Cell Biol 2009 ; 10 : 445-57.

7. Theveneau $\varepsilon$, Mayor R. Collective cell migration of epithelial and mesenchymal cells. Cell Mol Life Sci 2013 ; 70 : 3481-92.

8. Montell DJ, Yoon WH, Starz-Gaiano M. Group choreography: mechanisms orchestrating the collective movement of border cells. Nat Rev Mol Cell Biol $2012 ; 13: 631-45$.

9. Friedl P, Locker J, Sahai $\varepsilon$, Segall JE. Classifying collective cancer cell invasion. Nat Cell Biol $2012 ; 14: 777-83$.

10. Petitjean L, Reffay M, Grasland-Mongrain $\varepsilon$, et al. Velocity fields in a collectively migrating epithelium. Biophys J $2010 ; 98: 1790-800$.

11. Saez A, Anon $\varepsilon$, Ghibaudo M, et al. Traction forces exerted by epithelial cell sheets. J Phys Condens Matter 2011; 22 : 194119.

12. Tambe DT, Hardin CC, Angelini TE, et al. Collective cell guidance by cooperative intercellular forces. Nat Mater 2011 ; 10 : 469-75.

13. Theveneau $\varepsilon$, Mayor R. Cadherins in collective cell migration of mesenchymal cells. Curr Opin Cell Biol $2012 ; 24: 677-84$.

14. Theveneau $\varepsilon$, Mayor R. Can mesenchymal cells undergo collective cell migration? The case of the neural crest. Cell Adh Migr $2012 ; 5: 490-8$.

15. Weber GF, Bjerke MA, Desimone DW. A Mechanoresponsive cadherin-keratin complex directs polarized protrusive behavior and collective cell migration. Dev Cell $2012 ; 22$ : 104-15.

16. Kulesa PM, Teddy JM, Stark DA, et al. Neural crest invasion is a spatiallyordered progression into the head with higher cell proliferation at the migratory front as revealed by the photoactivatable protein, KikGR. Dev Biol $2008 ; 316: 275-87$.

17. Carmona-Fontaine C, Theveneau $\varepsilon$, Tzekou A, et al. Complement fragment C3a controls mutual cell attraction during collective cell migration. Dev Cell $2011 ; 21: 1026-37$.

18. Saragosti J, Calvez V, Bournaveas N, et al. Mathematical description of bacterial traveling pulses. PLoS Comput Biol $2010 ; 6$ : el000890.

19. Kriebel PW, Barr VA, Rericha $\varepsilon C$, et al. Collective cell migration requires vesicular trafficking for chemoattractant delivery at the trailing edge. J Cell Biol 2008 ; 183 : 949-61.

20. Inaki M, Vishnu S, Cliffe A, Rorth P. Effective guidance of collective migration based on differences in cell states. Proc Natl Acad Sci USA 2012; 109 : 2027-32.

21. Wang $X, \mathrm{He} L, W u Y I$, et al. Light-mediated activation reveals a key role for Rac in collective guidance of cell movement in vivo. Nat Cell Biol 2010; $12: 591-7$.

22. Emery G, Ramel D. Cell coordination of collective migration by Rabll and Moesin. Commun Integr Biol $2013 ; 6$ : e24587.

23. Ramel D, Wang $X$, Laflamme $C$, et al. Rabll regulates cell-cell communication during collective cell movements. Nat Cell Biol $2013 ; 15$ : 317-24.

24. Majumder P, Aranjuez G, Amick J, McDonald JA. Par-1 controls myosin-II activity through myosin phosphatase to regulate border cell migration. Curr Biol $2012 ; 22$ : 363-72.

25. Theveneau $\varepsilon$, Marchant L, Kuriyama S, et al. Collective chemotaxis requires contact-dependent cell polarity. Dev Cell $2010 ; 19: 39-53$.

26. Carmona-Fontaine C, Matthews HK, Kuriyama S, et al. Contact inhibition of locomotion in vivo controls neural crest directional migration. Nature 2008 ; 456: 957-61.

27. Xu X, Francis R, Wei C), et al. Connexin 43-mediated modulation of polarized cell movement and the directional migration of cardiac neural crest cells. Development $2006 ; 133: 3629-39$

28. David NB, Sapede D, Saint-Etienne L, et al. Molecular basis of cell migration in the fish lateral line: role of the chemokine receptor CXCR4 and of its ligand, SDFl. Proc Natl Acad Sci USA 2002 ; 99 : 16297-302.

29. Venkiteswaran G, Lewellis SW, Wang J, et al. Generation and dynamics of an endogenous, self-generated signaling gradient across a migrating tissue. Cell $2013 ; 155: 674-87$.

30. Dona $\varepsilon$, Barry JD, Valentin G, et al. Directional tissue migration through a self-generated chemokine gradient. Nature 2013 ; $503: 285-9$.

31. Reffay M, Parrini MC, Cochet-Escartin 0 , et al. Interplay of RhoA and mechanical forces in collective cell migration driven by leader cells. Nat Cell Biol 2014 ; 16: 217-23.

32. Reffay M, Petitjean L, Coscoy S, et al. Orientation and polarity in collectively migrating cell structures: statics and dynamics. Biophys J 2011; 100 : 2566-75. 


\section{RÉFÉRENCES}

33. Szabo B, Szollosi GJ, Gonci B, et al. Phase transition in the collective migration of tissue cells: experiment and model. Phys Rev E Stat Nonlin Soft Matter Phys $2006 ; 74$ : 061908.

34. Dumortier JG, Martin S, Meyer D, et al. Collective mesendoderm migration relies on an intrinsic directionality signal transmitted through cell contacts. Proc Natl Acad Sci USA 2013; 109 $16945-50$.

35. Theveneau $\varepsilon$, Steventon B, Scarpa $\varepsilon$, et al. Chase-and-run between adjacent cell populations promotes directional collective migration. Nat Cell Biol $2013 ; 15: 763-72$

36. Sanz-Moreno V, Gaggioli C, Yeo M, et al. ROCK and JAKl signaling cooperate to control actomyosin contractility in tumor cells and stroma. Cancer Cell $2011 ; 20: 229-45$.

37. Romanczuk P, Couzin ID, Schimansky-Geier L. Collective motion due to individual escape and pursuit response. Phys Rev Lett 2009; 102 : 010602.

38. Buhl J, Sumpter DJ, Couzin ID, et al. From disorder to order in marching locusts. Science 2006 $312: 1402-6$.
39. Théveneau $\varepsilon$. Migrations collectives de cellules mésenchymateuses. Med Sci (Paris) $2012 ; 28: 360-2$.

40. Reffay M, Parrini MC, Cochet-Escartin 0, et al. Migration collective : un partage des tâches entre cellules leaders et coordination supracellulaire. Med Sci (Paris) $2014 ; 30: 736-8$

41. Théveneau E. Chorégraphie sensuelle. Med Sci (Paris) 2013 ; 29 : 963-4.

42. Albrengues J, Meheguzzi G, Gaggioli C. L'invasion des cellules tumorales. Med Sci (Paris) $2014 ; 30: 391-7$.

43. Trepat $X$, Fredberg JJ. Plithotaxis and emergent dynamics in collective cellular migration. Trends Cell Biol $2011 ; 21: 638-46$.

TIRÉS À PART

$\varepsilon$. Theveneau

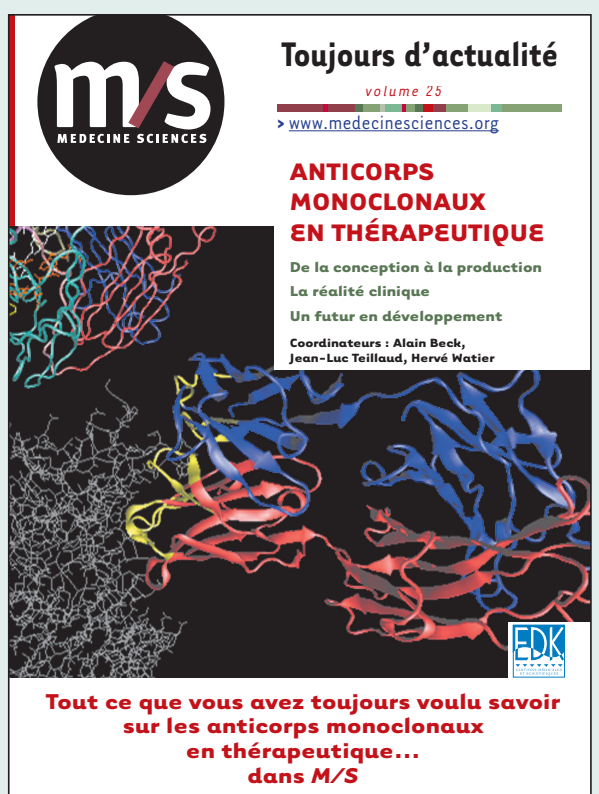

Tout ce que vous avez toujours voulu savoir sur les anticorps monoclonaux en thérapeutique... dans Médecine/Sciences. Pourquoi un numéro spécial de Médecine/Sciences sur les anticorps monoclonaux thérapeutiques? II nous a semblé que le moment était venu de dresser un état des lieux de ces biomédicaments qui prennent désormais une place considérable - et croissante dans les traitements de maladies souvent lourdes et désespérantes. Ce voyage que nous vous proposons à la découverte du monde des anticorps thérapeutiques nous a appris, ou plutôt rappelé, une évidence : les compétences en France sont fortes et nombreuses, qu'elles soient académiques ou industrielles, biotechnologiques ou cliniques. Le paysage français, trop longtemps discret, bruisse désormais de mille initiatives balayant de multiples aspects des anticorps thérapeutiques : études précliniques et cliniques menées avec de nouveaux anticorps dirigés contre des cibles originales, développement de nouveaux formats d'anticorps ou d'anticorps optimisés reposant sur des études structurales et fonctionnelles sophistiquées, recherche active de cibles pertinentes, mise au point de méthodologies de bioproduction, de couplage, etc. L'expansion industrielle rapide de ce champ est un défi que peut et doit relever notre pays, défi tant scientifique qu'économique, avec ses combats pour la propriété intellectuelle et pour l'emploi de nos jeunes scientifiques.

Alain Beck, Jean-Luc Teillaud, Hervé Watier

À retourner à EDK, 109, avenue Aristide Briand - 92541 Montrouge Cedex, France

Tél. : 0141177405 - Fax : 0143293262 - E-mail : edk@edk.fr

NOM :

Prénom :

Adresse :

Code postal :

Ville :

Pays :

Fonction :

Je souhaite recevoir $\mathrm{M} / \mathrm{S} \mathrm{n}^{\circ} \mathbf{1 2}$ - décembre 2009 (Anticorps monoclonaux en thérapeutique) : $25 €+3 €$ de port $=\mathbf{2 8} € \mathrm{TTC}$ en exemplaire, soit un total de $€$

Par chèque, à l'ordre de $\mathbf{E} \mathbf{D} \mathbf{K}$

Carte $n^{\circ} \mid \begin{array}{llllllllllllllllll}1 & 1 & 1 & 1\end{array}$

Date d'expiration : l 1 । 1 । 1 । $\quad N^{\circ}$ de contrôle au dos de la carte

Par carte bancaire :

Visa

Eurocard/Mastercard

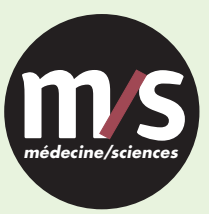

Tarifs d'abonnement $\mathrm{m} / \mathrm{s}-2014$

$>$ Grâce à $m / s$, vivez en direct les progrès des sciences biologiques et médicales

Abonnez-vous

à médecine/sciences

\section{Bulletin d'abonnement} page 813 dans ce numéro de $\mathrm{m} / \mathrm{s}$

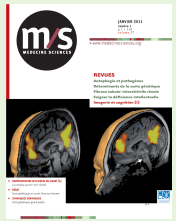

